\title{
111 ARMORED CAR T CELLS SECRETING 4-1BB LIGAND CROSSLINKED TO PD-1 CHECKPOINT INHIBITOR FOR ENHANCED SOLID TUMOR EFFICACY
}

Zachary Dunn*, Yun Qu, Melanie MacMullan, Xianhui Chen, Gunce Cinay, Pin Wang. University of Southern California, South Pasadena, CA, USA

Background Chimeric antigen receptor (CAR) T cell therapy has transformed the treatment of hematological malignancies but has yet to achieve similar success in solid tumors due to a lack of persistence and function in the tumor microenvironment. We previously reported the augmentation CAR $\mathrm{T}$ cell therapy in an engineered solid tumor model through the secretion of anti-PD-1 scFv, as shown by enhanced CAR T cell antitumor efficacy, expansion, and vitality. ${ }^{1}$ We have since matured the platform to create a superior cellular product CAR T cells secreting single-chain trimeric 4-1BB ligand crosslinked to anti-PD-1 scFv ( $\alpha$ PD1-41BBL). 4-1BB signaling promotes cytotoxic $\mathrm{T}$ lymphocytes proliferation and survival but targeting $4-1 \mathrm{BB}$ with agonist antibodies in the clinic has been hindered by low antitumor activity and high toxicity. CAR T cells using 4-1BB endodomain for costimulatory signals have demonstrated milder anti-tumor response and longer persistence compared to CAR T cells costimulated by CD28 endodomain. We have, for the first time, engineered CAR $\mathrm{T}$ cells to secrete a fusion protein containing the soluble trimeric 41BB ligand.

Methods We hypothesized that crosslinking the current antiPD-1 scFv with 4-1BB ligand would provide additional benefits to CAR T cells and is potentially of translational value in the management of tumors resistant to PD-1 blockade due to lack of $\mathrm{T}$ cell function. Therefore, we engineered CAR $\mathrm{T}$ cells to secrete a novel immunomodulatory fusion protein consisting of anti-PD-1 scFv crosslinked to a single-chain format of trimeric 4-1BB ligand, in which three extracellular domain units of $41 \mathrm{BBL}$ are connected with polypeptide linkers. The CAR T cells were then characterized in vitro and subcutaneous tumor models.

Results In vitro and in vivo, CAR19. $\alpha$ PD1-41BBL $\mathrm{T}$ cells exhibited reduced inhibitory receptor upregulation, enhanced persistence and proliferation, and a less differentiated memory status compared to CAR T cells without additional 4-1BB:41BBL costimulation. Accordingly, CAR19. $\alpha$ PD1-41BBL T celltreated mice displayed significantly improved tumor growth control and overall survival. Spurred on by our preclinical success targeting CD19 as a model antigen, we produced mesothelin-targeting CAR $\mathrm{T}$ cells and confirmed the enhanced solid tumor efficacy and persistence of $\alpha$ PD1-41BBL secreting CAR T cells.

Conclusions Given the significantly better therapeutic efficacy of $\alpha$ PD1-41BBL expressing $\mathrm{T}$ cells over $\alpha \mathrm{PD} 1$ expressing $\mathrm{T}$ cells, we believe that it is of high translational value to adopt secretion of $\alpha$ PD1-41BBL fusion protein to improve CAR $\mathrm{T}$ cell solid tumor efficacy, especially given the large number of patients that are PD1/PD-L1 therapy resistant.

\section{REFERENCES}

1. Li S, Siriwon N, Zhang $X$, Yang $S$, Jin $T$, He F, et al. Enhanced cancer immunotherapy by chimeric antigen receptor-modified $T$ cells engineered to secrete checkpoint inhibitors. Clin Cancer Res 2017;23(22):6982-92.

http://dx.doi.org/10.1136/jitc-2021-SITC2021.111 